\title{
The economic agent as rule maker and rule user: Homo Sapiens Oeconomicus ${ }^{\star}$
}

\author{
Kurt Dopfer \\ Department of Economics, University of St.Gallen, Sandrainstrasse 21, 9010 St. Gallen, Switzerland \\ (e-mail: Kurt.Dopfer@unisg.ch)
}

\begin{abstract}
The paper discusses the micro foundations of evolutionary economics, introducing a unified concept of the economic agent as a rule maker and rule user. Based on recent findings of the neuronal, cognitive and behavioral sciences, Homo Sapiens Oeconomicus emerges as an alternative to Homo Oeconomicus. A taxonomy of rules distinguishing between cognitive, behavioral and blueprint rules and a set of theoretical propositions related to the structure and evolution of those rules are suggested.
\end{abstract}

Keywords: Micro foundations of economics - Homo Sapiens Oeconomicus - Evolution - Rules - Cognitive, behavioral and neuronal economics

JEL Classification: A 12, B 41, B 52, B 53, D 00, D 64, D 80, D 83, E 11, L20

It is a pleasure and a privilege to contribute an article to the issue of this journal in honor of Professor Mark Perlman, whose work has an almost encyclopedic scope and embraces major contributions to institutional economics, labor economics, constitutional economics, and to various strands of evolutionary economics and the history of economic thought (Perlman, 1996; Perlman and McCann, 1998; see

\footnotetext{
* The present paper had its origin in a research project on "The Interdisciplinary Foundations of Economic Decision Making" supported by the Swiss National Science Foundation during 1978-1980. The core of that work was a 'brain model' whose architecture also serves as a corner stone for this paper. Since then there have been substantial developments in the respective areas; some of the major findings of the more recent research in the neuronal, cognitive and related sciences are discussed and integrated into the present paper. - I gratefully acknowledge insightful comments and criticisms from Georg D. Blind. Some ideas were discussed during my tenure as a Visiting Professor in the Department of Economics at the University of Queensland, in fall 2003, and I wish to record my appreciation of the hospitality during that visit. Particular thanks for the inspiring discussions go to Peter Earl, John Foster, and, as always, Jason Potts. Special thanks are due to Juli Lessmann and Charles R. McCann for their thoughtful corrections of my English prose and their editorial help. The usual disclaimer applies.
} 
also the publication in honor of Mark Perlman edited by Lim, Park and Harcourt, 2002). However, there is a perennial theme in Perlman's work, be it in institutional economics or the history of economic thought, a theme that establishes a unity in all that variety: it is the human individual. As any reader of his work will certainly acknowledge, this theme involves far more than the usual cliché that the human factor is important. This contribution is in the spirit of Perlman's work.

\section{Instrumental realism}

The following is an attempt to contribute some ideas on the problem of the micro foundations of economics from an evolutionary perspective. There is no general agreement within the discipline of economics about whether improved micro foundations will actually lead to better theory.

We share the view that more empirical groundwork, including interdisciplinary research, may indeed contribute to an improved reconstruction of theory. The following discussion explicitly recognizes the significance of the postulates of methodological realism. This position, however, does not endorse the view that there is virtue per se in gathering empirical data or engaging in interdisciplinary research. Any observational or experimental data are bound to meet the specific demands of a particular process of theory formation. The facts never speak without the instrumental guidance provided by some deductive notion. The advantage of an instrumentalist stand is that it presupposes a statement about the theoretical positions to which the micro foundations apply. Our approach to realism may be called instrumentalist in the sense that it calls for a clear deductive statement about the objectives of empirical research. Our methodological stand may therefore be called instrumental realism.

It is important to understand that there is an essential difference between the theoretical questions and the ontological underpinnings of neoclassical economics and evolutionary economics. The theoretical focus of neoclassical economics is static: it deals with the allocation of scarce resources in an equilibrium framework. In a neoclassical model, endogenous variables are always rapidly changing variables. The model relegates the comprehensive set of slowly changing variables to the exogenous realm. By contrast, evolutionary economics deals essentially with slowly changing variables. What is exogenous in the neoclassical model in fact comprises the endogenous core of evolutionary economics. In this sense, the broad scope of evolutionary economics constitutes a return to classical economics. The latter, too, made such variables as technology, institutions or preferences central to the model. In evolutionary economics, as in classical analysis, stasis is only a "time slice" through an inherently open and dynamic process.

Furthermore, neoclassical economics relies on a nomological approach. As in classical physics, a law is propounded which allows an event to be determined precisely, given sufficient information about initial and subsidiary conditions. The locus of the law in neoclassical economics is homo oeconomicus. The initial conditions are defined as resource constellations that offer the agents a set of profitable transactions. The set of subsidiary conditions given by the exogenous variables is 
kept constant. The terminal state is defined by an equilibrium where all profitable transactions have taken place and a Pareto optimum has been attained.

Let us consider the difference between neoclassical and evolutionary economics from an ontological perspective. The bare bones of this perspective are that all entities, agents, traded resources can be conceived of as carriers of rules. This view is derived from an ontology that we have developed more systematically elsewhere (Dopfer and Potts, forthcoming). A rule will be defined as a deductive schema that allows operations. The economic system is, in this view, seen as being composed of two levels: the generic (rule) level and the operant (production, transaction, consumption) level. In terms of a causal schema, the variables at the generic level are the causal ones, while operations are the effects or the consequences of these in the two-level schema. From the ontological perspective, the difference between neoclassical and evolutionary economics lies in their distinctive treatments of generic variables (for detailed discussion Dopfer, 2004). More specifically, the difference between neoclassical and evolutionary economics manifests itself in two ways. On the one hand, neoclassical economics deals only with a single generic variable represented by the behavioral rule disposition of homo oeconomicus, while evolutionary economics includes a broad range of generic variables. On the other hand, neoclassical economics keeps this rule variable constant, while evolutionary economics expressly allows for a change in rules.

The tenet of instrumental realism is that micro foundations must be helpful for theory construction and should not be remote from economic reality. Thus evolutionary economics calls for an economic agent who deals with rules in various complex ways.In an evolutionary perspective, homo oeconomicus is a rule-making and rule-using animal. The quest to recognize this nature of an economic agent does not stem here from the postulate of adequate realism. It results instead from the need to have instrumentally adequate micro foundations for evolutionary economics.

\section{Homo Sapiens Oeconomicus (HSO)}

The art of acquiring adequate micro foundations involves reconciling the demands of instrumental adequacy with those of empirical validity. In coping with the latter, the question arises of which empirically valid image of the human factor could serve as a yardstick for sufficient realism. We argue that homo sapiens should be the empirical reference point for any discussion about the realism of the micro foundations of economics. Evolutionary biology, anthropology and related sciences provide ample empirical material which allows us to formulate hypotheses or reasonable conjectures about homo sapiens. Homo sapiens appears, above all, to be an animal who uses and makes tools. Furthermore, homo sapiens exceeds all other primates in self-perception and the use of symbolic-verbal language. Human language, which has enabled efficient and efficacious social communication and has profoundly increased human cognition, has possibly co-evolved with the aptitude for tool making and tool using.

What is the general feature of homo sapiens? Tool production, tool usage, language creation, language use and related activities of homo sapiens have a common denominator in that they all rely on rules. Homo sapiens can thus be viewed more 
generally as a rule-making and rule-using animal. As a concept, homo sapiens obtains its instrumental adequacy when it operates in an economic context. Therefore, a construct that may serve as an appropriate micro foundation for an evolutionary economic theory is Homo Sapiens Oeconomicus (HSO).

The traditional homo oeconomicus fails in crucial ways with respect to the demand for realism. All available interdisciplinary evidence points to a picture of the human species in which this species deals in various and essential ways with rules. Any theory that involves human action but excludes the whole complex of rules, is likely to mis-state the nature of reality, not only marginally, but in a fundamental way. The central empirical deficit of homo oeconomicus is, therefore, not the unrealistic behavioral assumptions (confining decision making to a rational procedure), but the failure to take account of the basic nature of humans as rulemaking and rule-using creatures.

\section{Taxonomy of rules}

'Rule' and 'rule carrier' are analytical terms that can be employed in various theoretical contexts. In biology, rules are genes, and rule carriers are organisms or species. The beauty of the neo-Darwinian model is that the entire process of biological evolution can be expressed on the basis of a single rule-type - the gene. The question of particular interest at this juncture is whether there is analogously in economics a single rule in the form of an 'economic gene' that would allow us to expound the salient characteristics of the evolutionary dynamics and structure of an economic system. While reduction is the hallmark of any scientific inquiry, we argue that economic evolution is more complex than biological evolution and that any reduction to a single rule-type would mis-state essential aspects of the subject matter under investigation.

We propose a rule taxonomy that embraces three rule types with distinct rule carrier instances: cognitive, behavioral and blueprint rules. Though closely interrelated, these rule types cannot be substituted for each other; the process of cognition, for instance, must be explained by cognitive rules and cannot be explained by any other rule type. The rule taxonomy presented in Figure 1 introduces the notions of agent rules and organizational rules, recognizing the significance of the distinction between the agent and the environment of which it is a part. The general distinction is useful since most evolutionary models are built on the premise of a systemic agent-environment relationship.

Cognitive rules refer to cognition, or thought processes, which are independent of the external world and of observation. The reality evoked by cognitive rules is timeless and spaceless and enables us to solve economic problems by reconstructing the past and by imagining possible futures. There is a growing body of studies in cognitive economics which allows expressly for the integration of the evolutionary dynamic of cognitive rules (Rizello, 2003). In this vein, Dosi et al. (2004) have argued that "cognitive rules or categories (....) go together with various mechanisms of framing through which information is interpreted and also rendered operationally meaningful to the decision-makers.(....) Frames appear to be (....) an ubiquitous feature of both decision-making and learning. What one understands is filtered 


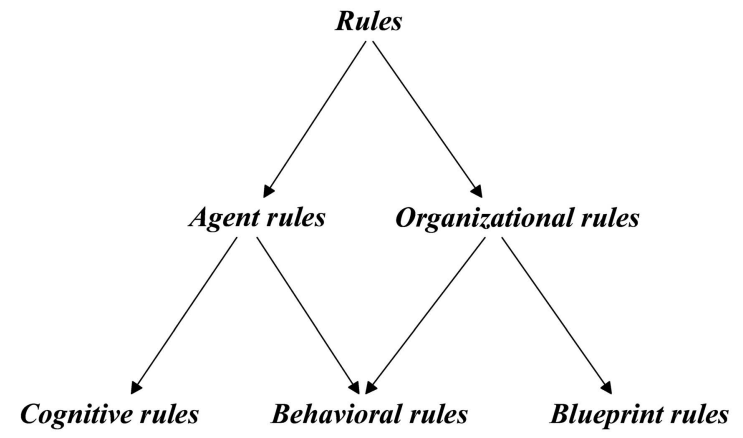

Framing devices, mental models, learning models, heuristics, algorithms
Individual behavior: Rule creation and adoption Collective behavior: Frequency dependence, behavioral parameter
Artefacts: Rules for objects Organizational architecture, production technology, product design, machines, equipment

Fig. 1. Taxonomy of rules

by the cognitive categories that one holds and the repertoires of elicited problemsolving skills depend on the ways the problem itself is framed."

Individual behavior, like cognition, is governed by the human cortex. The subsequent discussion that revolves around Figure 2 will highlight the connections between behavior and various cortical areas. In contradistinction to cognition, however, individual behavior also always relates in some way to an instance of the external world. Individual behavior is a joint result of internal cognitive powers and external environmental contingencies. Externally, individual behavior is governed by a double contingency. First, it relates to the behavior of other agents, and second, it relates to objects, such as artefacts and physical resources. External behavioral referents are always associated with the task of organization. The coordination of the behavior of many agents requires rules for social organization, while the allocation of physical resources requires rules for technical organization.

Organizational rules apply to all levels of an economic system. At a micro level, transaction rules enable exchange operations in the market, and/or the organizational rules of a firm its productive operations. At a macro level, organizational rules refer to the coordination of market behaviors and the allocation of the traded resources. At all levels, the organization represents a composite of individual rules to which the agents must submit. The question is not whether agents in an organization must submit to rules but rather who, or what, authority sets those rules. The micro and the macro levels deal with different degrees of organizational complexity. In a market economy (unlike in a centrally planned one), the organization of the micro units, such as the firm, is trusted to the autonomy of individual agents, such as the entrepreneur, while, at the macroscopic level, coordination and allocation are left to the self-organizing powers of the market. The discussion in this paper is on the micro foundations and invites a focus on intentional behavior and consciously planned forms of organization.

In this perspective, the firm is a complex nexus of organizational rules which vary in their ability to coordinate the behavior of its agents and to allocate its 
resources. (The division between ability-based and resources-based approaches is ill conceived). The organization of physical objects, such as machines or technical equipment, can be based on fixed templates, and in our taxonomy we refer to this type of rule as a blueprint rule. In their regulatory function, the rules pertaining to social organization resemble blueprint rules, but in relation to human behavior they do not share the determinism of the blueprint rules as this stems from the logic of technology. The explanatory core of an evolutionary theory of the firm or any other micro unit must be a behavioral model that links with both the subjective cognitive dimension of rules and the objective blueprint dimension (Sect. 4).

It is possible to explain the structure and dynamics of an economic system in a mono-causal way by focusing on one rule dimension only. In a Marxian perspective, for instance, the objective laws of the aggregate technical rules determine the aggregate social rules the objective interplay of which governs the structure and long-run dynamics of an economic system. While cognitive rules lose their meaning in the deterministic machinery of the Marxian model, they play a pivotal role in Austrian economics and related subjectivist strands of the discipline. From an evolutionary point of view, the structure and dynamics of an economic system can be explained only on the basis of a model that embraces all three dimensions - the cognitive, behavioral and blueprint rule dimensions.

\section{Rule trajectory}

Besides rule and rule carrier, a third term that highlights the process dynamic of a rule will be introduced. In biology, a new rule is created by an error in the genetic replication of a whole organism, and evolution occurs when that error is selectively retained in a species over generations. In economics, micro units, such as firms, that are carriers of "economic genes", are rarely replicated as wholes. Instead, partial rules are generated and adopted macroscopically by a population of carriers of this kind. The Darwinian schema of mutation-selection-retention operates with implications that do not apply to economic evolution, and a more general schema of the rule dynamic is therefore required in order to arrive at a valid theoretical exposition of economic evolution. A rule trajectory that expounds the essential general features of rule evolution can be stated in terms of three process phases:

\section{Origination of rule}

2. Adoption of rule

3. Retention of rule

The first phase feeds on cognitive rules, and the trajectory process is set in motion when a novel rule (invention) is connected to the behavioral domain (innovation). The core of the second phase represents a path-dependent dynamic in which an existing adoption frequency influences subsequent adoption probabilities. An organizational rule may be introduced intentionally into a firm's domain, but its implementation will depend on the power of the emergent behavioral parameter. In the third phase, a critical adoption frequency is reached and the collectively adopted rule is used recurrently for economic operations. The self-stabilizing retention pro- 
cess of the third phase can be labeled an institution. The routines that Nelson and Winter (1982) wrote about are rules that have attained institutional status.

\section{Biological predisposition}

So far, we have sketched a model which views the cognition and behavior of HSO as governed by its previously acquired rule disposition. This was based on the assumption that HSO is equipped with a biological predisposition which enables the formation of a distinct disposition for how to behave and think in the economic context. The notion of biological predisposition is broader than that of generic (culturally contingent) disposition. Homo sapiens has a phylogenetic history, and the biological predisposition had been in existence long before the generic one was acquired. A salient characteristic of biological evolution is that antecedent life-forms are not lost, but rather are integrated into the higher, more complex, life-forms.

The human brain is thus composed of both phylogenetically old and phylogenetically new neuronal mechanisms. We denote the former as the archecortex, and the later as the neocortex. The distinction is employed in both evolutionary biology and the neurosciences (Zeier, 1980). We take over the general empirical content of the concepts from these disciplines, but use them in a way that enables them to still serve the purpose of economic theory formation. We define the archecortex as a complex of interconnected neuronal mechanisms that perform a range of tasks that can be accomplished on the basis of automatisms. As these cortical mechanisms are not acquired by learning, they are independent of the rule disposition. The neocortex accommodates more complex mechanisms, which include the faculty to form a rule disposition that enables complex problem solving.

Figure 2 provides a conceptual model which delineates the brain in its neuronal interconnectivities between archecortex and neocortex (vertical circular loop) on the one hand, and the specific sets of tasks which the cortical domains can perform (horizontal circular loops) on the other. The archecortical mechanisms perform various internal basal functions that relate to the respiration system, blood circulation and metabolism. The neocortical mechanisms enable complex operations that relate internally to cognition and externally to behavior aimed at complex problem

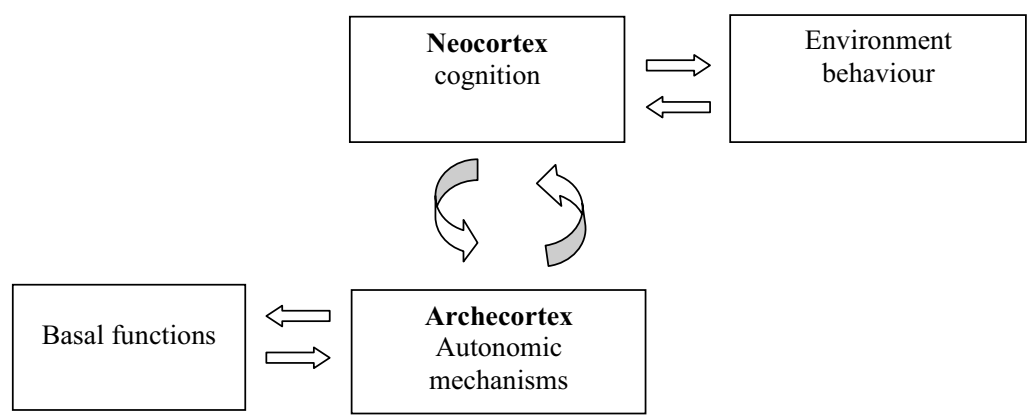

Fig. 2. Model of brain with internal and external loops 
solving in evolving environments. The upper horizontal loop essentially denotes the formation of the rule disposition of HSO.

Cortical interconnectivity means that the archecortex influences, via its automatisms, higher cognition and behaviors. In turn, the neocortex exerts a limited conscious control over the auto-mechanisms. The link from the phylogenetically old areas to the neocortical domains is of particular interest, since economics naturally deals with complex problem-solving involving advanced areas of the cortex. Emotions, moods and feelings are archecortical manifestations that may significantly influence neocortical functions (Damasio, 2000), such as cognition and behavior in economic contexts. These "non-rational manifestations", as they are conceived in mainstream economics, may be subject to conscious control, but the essential point to grasp here is that emotions, moods and feelings are always present in the cortical disposition governing economic actions. They represent potentially powerful, and mostly unpredictable, forces in the economic process. We shall return to the issue of neuronal interconnectivities in a moment when discussing characteristics of the neocortex.

\section{Neocortex: on cognition}

The hemispheric specialization of the neocortex is an evolutionary improvement found uniquely in the human brain. The left and the right sides of the neocortex perform distinct functions (Sperry, 1974). The left hemisphere is specialized in analytical, sequential and propositional abilities required for verbal expression and arithmetical operations. It allows syntactical reasoning and computational procedures such as adding, multiplying or equation solving, to be carried out. The right hemisphere, by contrast, is specialized in geometric, synthetic, pictorial and spatial abilities. It enables the brain to combine elements into a whole, say to assemble colored blocks to make a mosaic picture (Taeuber, 1974, in Eccles and Popper, 1977), allowing a comparison of singular observations, images or conceptions to be made on the basis of a Gestalt. While the left hemisphere is positional, the right breaks up the whole into its parts. It is able to conceive isolated entities as complementary components of a whole and to comprehend the holistic nature of this whole. The division of knowledge and labor in an economy relies fundamentally on the ability of this type of relational and appositional thinking.

\section{Cortical fuzziness: on indeterminacy}

The brain is sketched as being composed of an archecortical and a neocortical area, with a division into specialized hemispheres of the latter. Temporarily, there can be a certain dominance in the neuronal activities pertaining to a specific compartment depending on the type of cognition or behavior involved at a particular time. However, as a most important feature of neuronal organization, the high thalamocortical interconnectivity, which represents a hub from which any site of the cortex can communicate with one or more other such sites, carries out the task of overall co-ordination and control (Llinas, 2003). In particular, the interconnected cortical 
levels allow the neocortical control of areas responsible for body-sensory functions and emotions to operate. Individuals have a cognitive ability to identify, understand and instrumentalize emotions adaptively and to use them as some form of intelligence (Foster, 2000). In connecting emotional intelligence to the requirements of an economic system, we are not dealing here with a "logical form of intelligence that corresponds to the IQ, but instead with an "evolutionary intelligence", which "reflects the ability to learn and change" (Allen, 2004). Higher order mental activities are embedded in neuronal structures of the archecortex and produce a soft emotionally colored cognition. The archecortical - particularly thalamic, cerebellar and hippocampic - areas interconnected with the neocortical site equip humans with a cortical organization that provides them with intelligent emotions (viewed from the archecortex) and with emotional intelligence (viewed from the neocortex).

Similar interconnectivities exist with respect to the lateralization of the brain. The left hemisphere adds to the performance of the right by providing some essential verbal symbolism and syntactical assistance. The right hemisphere, in turn, supports the left with its pre-eminent properties of spatial, relational and integrating abilities. There is no unilateral dominance of one hemisphere over the other, say the left over the right, but rather a complementary specialization. The hemispheric interconnectivities generate a basic fuzziness in human cognition, and we may assume that it is precisely this indeterminacy of the cognitive process that constitutes a major source of human creativity. The interplay of distinct, but complementarily specialized abilities triggers the imagination that is at the very root of the creation of novelty, and that, in turn, propels economic growth and development. Any economic theory designed to cope with economic change is bound to recognize the creative and imaginative sources of novelty, discovery and innovative problem solving.

\section{Consciousness}

The interconnected sites of the human cortex accommodate specific abilities. In addition, the unity of the cortex gives it a non-specific ability to conceive. (Edelman and Tononi, 2000). Where we should localize the cortical self-reflection or self-recognition that may be embraced by the notion of consciousness still seems to be an open issue in the literature of the neurosciences and philosophy. We propose that the domain of all cortical interconnectivities represents not only the locus of instrumentally specific cognitive abilities but also of non-specific human consciousness.

More specifically, consciousness allows for intentionality. Intentionality gains practical significance when it receives support from will power. This component of instrumental consciousness provides the base for individual economic operations, such as decision making and individual choice.

The factors of intentionality and will power are closely associated with the ego or the self of a person. Eccles and Popper have proposed a dualistic interaction theory where the conscious self interacts with the dominant left hemisphere of the brain. Their theory is useful for reconstructing the micro foundations of economics since it emphasizes the active role of the individual. However, it has been challenged on grounds that it is trapped in Cartesian Dualism (Bunge, 1980). The 
theory may also be challenged on neurophysiological grounds given that the right (and not the left) hemisphere with its holistic abilities could cope better with the problem of unity, presumably by conscious control or self-awareness. Given the double interconnectivities, the neuronal pattern of the conscious state may change, depending on the dominance of the neuronal activities of the cortical sites. Hence, both the intensity and the locus of cortical dominance may vary considerably, and this will give the human self its distinct character at a particular time. HSO has a bounded self as well as bounded rationality.

\section{Departures from homo oeconomicus}

The preceding discussion has been guided by the methodological imperative of realistic assumptions. The following propositions about HSO provide some substantive rationale for a critique of the fundamental shortcomings of neoclassical homo oeconomicus and may serve as an interdisciplinary platform for the subsequent discussion. The propositions are:

1. Non-decomposability of cortical levels and hemispheres, which govern human cognition and behavior,

2. Non-homogeneity of specialized cortical areas compounded by complex cortical double interconnectivities,

3. Non-periodicity of both dominant and fuzzy interconnected neural processes.

The proposition of non-decomposability strikes a fatal empirical blow to the unilateral model of homo oeconomicus. Computational and analytical abilities are emotionally colored, and they are linked simultaneously with the ongoing pattern generation of the lateral counterpart. Perfect rationality based on splitting the cortical hemispheres and the exclusive activation of the left is - given the findings of modern neuroscience - an abstruse concept. The neoclassical decomposability assumption not only yields an ill-conceived concept of rationality, but it also shuts all doors to an investigation of the sources of creativity and ingenuity in complex economic problem solving because it ignores the cross-cortical fuzziness.

Second, the proposition of non-homogeneity provides a point of departure for an analysis of the complex variety that governs economic cognition and economic behavior. Non-homogeneity, or variety, is an essential characteristic of all evolutionary approaches. There is a fundamental, ontologically-rooted, difference between typological thinking dealing with averages, on the one hand, and population thinking allowing for variety, on the other (Metcalfe, 2004). The neoclassical representative agent who appears as a machine à l'homme is entirely at odds with neurophysiological evidence.

Third, the proposition of non-periodicity of cortical activities has both theoretical and methodological implications for economics. Theoretically, the differences that occur with repeated economic actions are not anomalies but constitute the normal case. This is to say, variation in behavior is not an anomaly to be explained, but is instead the very essence of evolutionary rational behavior. Furthermore, given the circular causation between agent and environment (upper loop in Fig. 2), the intercortical fuzziness suggests the hypothesis that any external pressure exerted on 
an agent may generate novelty and a change in problem solving procedures. Environmental constraints feed back to the agent, and by spurring new problem solving, affects the extant constraints reversely. Standard verification relies on repeatability criteria, but this cannot be done in an evolutionary context. Instead, such a context must allow for non-repeatability, and thus accept heterogeneity and change as standards of normality.

\section{Operant and generic agenda}

In contemporary economics, we are witnessing a growing interest in empirical research related to the reconstruction of the micro foundations. A large body of data has been provided by research conducted in experimental behavioral economics and in neuroeconomics. These studies are generally interesting with regard to both the experimental and statistical methodologies employed and the way in which the experiments are interwoven with a theoretical schema. The theoretical questions addressed by drawing on recent behavioral and brain research are mainly inspired by neoclassical economics. Their kind of empirical research is designed to challenge the empirical status of homo oeconomicus assuming that neoclassical economists are generally concerned with the realism of assumptions.

A research program emerges that is very much committed to the "hunt for anomalies" of homo oeconomicus. All findings obtained so far demonstrate that humans are not merely rational decision makers who pursue selfish aims, but are as well creatures who systematically contradict the rationality assumption by widely exhibiting reciprocally altruistic behavior (e.g., Fehr and Gächter, 2002). In particular, the findings of experimental neuroeconomics demonstrate that economic transactions not only lead to the activation of brain areas responsible for cognitive processes, such as the prefrontal dorsolateral areas, but they also simultaneously activate areas such as the anterior insula which are the locus of emotions and typically archecortical functions (Sanfay et al., 2003; Camerer, 2003). Ongoing experimental studies approach the micro foundation problem from two different directions behavioral economics focuses on external behavior and neuroeconomics concentrates on the internal behavior of economic agents. The findings of both research areas yield a picture of an operant human agent who is both boundedly rational and emotional, selfish and reciprocally altruistic, fair and unfair, all at the same time.

The research conducted in the two fields does not address the question of the basic nature of the cognitive and behavioral dispositions. The program focuses on theoretical questions concerning regularities in economic operations and thus presumes a basically constant disposition. However, empirical research aimed at analyzing the generic level must start with the assumption that cognitive and behavioral dispositions change over time. The core of an evolutionary micro program consists of an analysis of generic change. The research questions are based on the premise that the cognitive and behavioral dispositions of economic agents are not, in general, original, they also involve to a significant extent a body of acquired, culturally contingent rules. 


\section{Towards micro foundations for evolutionary economics}

The instrumental adequacy of empirically warranted micro assumptions depends on the theoretical framework for which they are designed. It is beyond the scope of this article to outline a full blown theoretical agenda for evolutionary economics, but a few building blocks dealing with requirements for micro foundations may be furnished. The analytical core unit is defined as a rule which is actualized by the population of its carriers. We call this analytical unit a meso regime.

The macro domain of the economic system is viewed as a structure composed of meso regimes. The macroscopic structure refers to both the structure of rules and the structure of rule adoption frequencies. Thus, there is a deep structure that is composed of ideas or semantic information defining the rules, and a surface structure that refers to the relative frequencies of the physical rule-actualizations. Whereas the deep structure is invisible and can only be described qualitatively, the surface structure is visible, and thus measurable in numbers.

The analytical unit of a meso regime is neither micro nor macro. It includes individual agents as micro components, and is a complementary component of a macro structure. We argue that the meso unit is at the very heart of an evolutionary analysis designed to explain economic coordination and economic change. Considering the significance and possible explanatory power of the concept, it is surprising how little attention the branch of a so-defined meso-economics has received up to now. (Dopfer et al., forthcoming).

The question is this - how can the meso concept be used for developing a better micro-foundation, in particular in relation to cognition and behavior? The concept of a rule brings the issue of complementarity into focus. A rule must fit the structure of complementarities in a system. The individual cognition and behavior underlying the logic of rule complementarity is adaptation. Darwin's essential lesson shows that rules will not be selected if they are not adapted to the other rules (which compose the environment). The economic criterion to be met here is not efficiency, but efficacy. An agent can only be economically successful if equipped with a disposition that allows efficacious cognition and efficacious behavior. This applies to both factor markets, which call for an understanding of technical complementarities or the integration of routines into capital (Cantner and Pyka, 1998), and consumer markets, where preference complementarities and lifestyle adaptations are important (Earl and Potts, forthcoming).

Up to now, there has been little experimental evidence suggesting that special cortical areas are activated when economic agents perform tasks related to the solution of pattern problems. Experiments demonstrating that neuronal activities in special cortical areas do indeed occur during the process of adaptation to the complementarity requirements cited above would be an important step towards an empirically valid economic theory.

The preceding discussion has brought the aspect of economic structure into focus. However, each structural component of the economic system is, by its very nature, also dynamic. A structural component represents a process involving cognition and behavior. We have conceived the structural process unit as a rule that connects with other rules in a structure and unfolds as a process along a trajectory 
with three phases. Phase 1 is associated with the generation of novel rules. Phase 2 involves selective adoption and learning, phase 3 is characterized by stabilization and retention at both the microscopic and the macroscopic scales. What needs to be analyzed at this point is how cognition and behavior that are needed to perform phase-specific tasks may be explained on the basis of available empirical evidence. However, there is another important issue which we need to address first.

\section{Herbert Simon's concept of docility}

Humans have specific capabilities concerning the processing of rules. Likewise, their cognition and behavior can be rule-efficient or rule-efficacious to various extents. However, there is also a general biological predisposition which motivates humans to get involved in rule activities. Besides motivation, there must be a basic trust in the rule behavior of other agents and in the validity of rules generated by them.

Simon has argued that humans have a biological disposition for "docility" (2004). He suggests that humans generally do not consider costs or material rewards when producing rules for the public domain. At the generic level, economic agents display behavior characterized by non-reciprocal altruism. Simon criticizes the narrowing down of the concept of altruism to its reciprocal form. He specifically rejects any attempt to employ his own concept of bounded rationality in the context of reciprocal altruism as suggested by neo-institutional economics.

Rule altruism is effective whenever it encounters trust on the part of a potential rule adopter. This generic trust extends beyond the operant trust employed in market transactions. Much of the knowledge upon which economic agents operate is provided by other agents and a general absence of generic trust would lead to a breakdown of the entire system. (Noteboom, 2003; Khalil, 2003).

As a brief, but perhaps important, methodological note, I would like to remark that the received doctrine of experimental economics always suggests a search for behavioral regularities. For instance, empirical regularities in economic cooperation are derived from an experimental setting involving group selection. The hallmark of the procedure is that its empirical results are static because they are designed to demonstrate how reality is at some specific point of time. By contrast, evolutionary economists adopt a more process-oriented view. Rules are considered to be emergent properties of a rule adoption process occurring in a group. The dynamics may be modeled on the basis of the rule trajectory proposed earlier. Simulation models seem to be an appropriate tool for this task, as they allow different assumptions about the rule disposition of agents, and modeling the rule adoption process in its emergent, often unpredictable properties.

Assumptions of this kind are not a trivial matter, and there must be good arguments to warrant any work aimed at improving the empirical content of economic theory. We argue that homo sapiens oeconomicus is a rule-maker, and this includes the proposition that the dispositions of the agents, which determine their cognition and behavior in economic contexts, evolve over time and feature emergent properties. Let us see how the unique evolutionary accomplishment - the sapiens - merges into homo oeconomicus. 


\section{Beyond monkeys}

What can homo sapiens do that other primates cannot? (Note 'primates' refer to members of the highest order of mammalia including humans, monkeys, lemurs etc.) Humans can, as we know, use tools. This is a remarkable evolutionary achievement, but not unique to humans. Various experiments have shown that other primates demonstrate surprising aptness when exposed to problems whose solution call for cognitive skills, for instance, using sticks to collect ants. Furthermore, homo sapiens is capable of learning but so too are other primates. For instance, observations of macaque populations on the Japanese island of Koshima show that skill in washing potatoes in the ocean has spread over the entire population (Kawai, 1965; in Zeier 1980). The skill has been observed, understood as a rule, and then applied as such.

Humans are typically social animals; but so are most other primates and many other species as well. A large part of the social behavior of primates is due to nonacquired original behavior, but recent observations have shown that the biological predisposition also allows a generic rule disposition of social behavior to be formed. For instance, rhesus monkeys are notorious for their aggressive nature while stumptail monkeys typically show cooperative and conciliatory behavior within their group. Co-housing the juveniles of the two species leads to a dramatic change in their behaviors: the rhesus monkeys co-housed with the stump-tail macaques adopt the cooperative and conciliatory behavioral rules of the latter (de Waal and Johanowicz, 1993; in Cummins, 2001).

Hope that the human species scores better with regard to its uniqueness does seem to be warranted when we turn to culture. Humans have the ability to preserve rule-knowledge over generations, and in doing so they have history. However, the fact that evolutionary anthropologists have recently discovered a workshop for nut cracking in the Tai forests of the Ivory Coast that is 150 years old (Boesch, 1999) is disappointing if we want to think of our species as unique. The "tool capital" of the workshop and the tool knowledge have been handed down from generation to generation. We usually associate such activities with culture and history.

So, what does make humans unique? It is imagination. It is the ability of the human brain to entertain a world view that no longer relies exclusively on experience but also on images created "inside" the cortex. This implies the freedom to imagine a future that is disconnected from the past. Humans are imaginative rule-makers.

Let us consider the production of tools as an exemplar. Primates have combinatorial skills, and, given their inherited instinct to play, some kind of chance events that lead to the use of tools are likely to occur frequently. Chance events still play a large role in all human invention, but unlike other primates, humans recognize the regularities that show up in the process of trial and error and see them as a complex of rules. Humans are able to plan the chance process and devise their rules accordingly. Humans make technical rules. Even today, the conscious use of trial and error in discovery is still very important in some industries, for instance, the biochemical and pharmaceutical industries.

Humans are capable of socially and technically organizing their conscious efforts to increase their knowledge base for economic operations. Individual imagination occurs in a context of socially shared imagination. This creative circuit 
essentially distinguishes human life from the social life of other primates who respond not to cognitively-anticipated problems but to environmentally-given ones.

Symbolic-abstract language may have played a crucial role in the emergence of shared imaginary worlds. Human culture was possibly triggered by the change in a single gene that relates to the larynx and tongue and was necessary for evolving speech and verbal language. Küppers has argued that the essential difference between humans and all other nature lies in the fact that humans can ask questions and the rest of nature cannot (Küppers, 1990). This is not just a metaphor, as this ability accounts for the essential fact that humans can exercise their imagination.

Arguably, the concept of imagination has not exercised that of mainstream economists. However, from the time of classical economics onwards there have always been economists who did recognize the significance of this human trait for economic life. More recently, the proponents of post-Keynesian and Austrian economics have given primacy to the concept of imagination in their work. For instance, Shackle put the notions of imagination and radical uncertainty at the center stage of his post-Keynesian analysis (Shackle, 1981), and Loasby recognizes the significance of creative advance for the process of the growth of economic knowledge (Loasby, 1999). Witt has employed the concept of imagination in his theory of the firm and has introduced the notions of vision and cognitive leadership (Witt, 1998). Metcalfe evokes human imagination in his concept of "restless capitalism", which is unique in its ability to explain the task of coordination in the "restless" context of experimenting and innovating (Metcalfe, 2004). In his earlier work, Hayek developed a neuronal network model of the brain and connected it to the demand for, and capability of, alertness and imagination that individual agents are confronted with in their search for information in a decentralized market (Hayek, 1952). Following Hayek's lead, various authors have further developed the neuronal-cognitive network model (Herrmann-Pillath, 1992; Birner, 1999; Feser, 2003).

There is little evidence available for where and how imagination occurs in the human brain. Neuroeconomic experiments may be more difficult to conduct, since the phenomenon of imagination is conceivably less amenable to analytical precision than that of the emotional articulation of fear, anger or aversion. However, the significance of the factor of imagination for economic life can not be discounted on grounds of experimental or methodological difficulty.

\section{Rule adoption: efficiency}

The second and third phases of the trajectory refer to the various forms of rule adoption. (Note the difference between adoption and adaptation, which is one form of adoption). It begins with the complex decision to adopt a given rule (Winter, 2004), to learn it, to adapt it to fit into the existing knowledge base of the agent in phase 2 (Day, 1975; Dosi et al., 2004), and finally to stabilize the adopted rule in the knowledge base for recurrent use in phase three (Nelson, 2002). The criterion applied in rule adoption is basically one of efficiency. The measurement rod is basically quantitative. Given its efficacious application, the adoption of a rule may be more or less efficient. 
From an evolutionary angle, it will be recognized that efficiency may apply to either the generic or to the operant level of an economic system. The latter is the domain of neoclassical economics; specifically, of efficiency stated in terms of optimum decision making under constraints. The performance standard is operant efficiency applied in a decision theoretic context. In contradistinction, cognition and behavior along the rule trajectory refer to generic traits and consequently the performance standard is generic efficiency. That is, generic cognition and generic behavior may be more or less efficient. A learning rule $A$ may be efficiency-superior to learning rule $B$, or the generic knowledge base of an agent $X$ may enable that agent to employ rule $A$ more efficiently than agent $Y$. Analogously, generic efficiency is a relevant criterion in phase three when maintaining the knowledge base of a rule carrier. Agents or firms will have a selective advantage in a competitive market if they can base their operations on superior routine-efficiency.

Localization of the cortical areas that account for the ability of efficient cognition and efficient behavior seems to be straightforward on the basis of the brain model outlined. The tasks in question require analytical and sequential skills typically performed by the left hemisphere of the cortex. Neuroeconomic experiments presumably will demonstrate that increased neuronal activity arises when a subject is asked to solve an analytical, propositional or sequential problem.

However, the experimental outcomes may differ depending on whether the requirement for efficiency relates to the performance of an operant or to a generic task. Calculations when shopping, drawing up a financial business plan, using a pocket calculator, abacus or supercomputer commonly require abilities for solving analytical and sequential tasks. In turn, rule adoption and learning involve a complex procedure for fitting new knowledge into a given knowledge base. Generic efficiency calls not only for computational skills but also for abilities that rely on the neuronal activities of the right hemisphere of the cortex. Additionally, it will also involve a larger measure of motivation which depends on the archecortical layers responsible for emotions and drives. As with imagination and efficacy, the measuring of generic efficiency in an experiment will prove to be a challenging task due to the cortical interconnectivities above mentioned. The link between economic behavior and the activated cortical areas may be fuzzy, but it does exist and may be further investigated scientifically.

\section{Conclusions}

Ever since economics emancipated itself from philosophy in the second half of the $18^{\text {th }}$ century, it has been occupied with two grand questions: economic coordination and economic change. As we have seen, neoclassical economics makes the first question its focal point and views the dynamic as a path of continuously restored equilibria. Coordination is interpreted as the allocation of scarce resources under the assumption of a law (rationality assumption) and a ceteris paribus clause with respect to the slowly changing historical variables (technology, preferences, institutions, and other generic variables). Micro foundations for neoclassical economics refer to the rationality assumption and to the related domains of operant 
cognition and operant behavior. We have acknowledged recent progress in experimental behavioral economics and neuroeconomics. But we have also challenged the view that empirical findings of an experimental program centering around these questions will automatically produce a new (and possibly non-) neoclassical theory.

The research program of evolutionary economics differs from that of neoclassical economics in two essential ways. First, it starts expressly with dynamics and views coordination as a temporary stationary state of an evolving economic system. Second, the explanation of coordination and change takes place at the generic level defined as the locus of economic rules. The micro foundations of an evolutionary economic theory must meet the criteria of instrumental adequacy in terms of understanding the cognition and the behavior of homo sapiens oeconomicus as a rule maker and a rule user. Economic performance is therefore not confined to operant efficiency, such as allocation efficiency; it includes generic efficiency and efficacy as well. The forces at work in an evolving economic system, and the standards by which their performance is measured, are captured by the triad of factors: imagination, efficacy and efficiency.

The construel of homo sapiens oeconomicus is designed to frame relevant questions for an evolutionary economic theory and to furnish some empirically acceptable assumptions for its micro foundations. We close with a note of hope - the hope that experimental economists and modelers of all kinds will gradually occupy the terrain and close the remaining conceptual and empirical gaps.

\section{References}

Allen P (2004) Economics and the science of evolutionary complex systems. In: Dopfer K (ed) The evolutionary foundations of economics. Cambridge University Press, Cambridge

Birner J (1999) The surprising place of psychology in the work of F.A. Hayek. History of Economic Ideas 7(1-2): 43-84

Boesch Ch (1999) Hunting tools, and sociality: a new evolutionary scenario for chimpanzees and humans. International Symposium on The Evolution of Apes and the Origin of Human Beings, Inuyama, Japan

Bunge M (1980) The mind-body problem: a psychobiological approach. Pergamon, New York

Camerer CF (2003) Strategizing in the brain. Science 300: 1673-1675

Cantner U, Pyka A (1998) Technological evolution - an analysis within the knowledge-based approach. Structural Change and Economic Dynamics 9: 85-107

Cosmides L, Tooby J (1996) Better than rational: evolutionary psychology and the invisible hand. The American Economic Review 84(2): 327-332

Cummins DD (2001) The impact of the social environment on the evolution of mind. In: Holcomb III HR (ed) Conceptual challenges in evolutionary psychology. Innovative research strategies. Kluwer, Dordrecht

Damasio AR (2000) The feeling of what happens: body and emotion in the making of consciousness. Harcourt, New York

Day RH (1975) Adaptive processes and economic theory. In: Day RH, Groves T (eds) Adaptive economic models. Academic Press, New York

Dopfer K (2004) Evolutionary economics: a theoretical framework. In: Dopfer K (ed) The evolutionary foundations of economics. Cambridge University Press, Cambridge

Dopfer K, Potts J (forthcoming) Evolutionary realism - a new ontology for economics. The Journal of Economic Methodology

Dopfer K, Foster J, Potts J (forthcoming) Micro - Meso - Macro. Journal of Evolutionary Economics 
Dosi G, Marengo L, Fagiolo G (2004) Learning in evolutionary environments. In: Dopfer K (ed) The evolutionary foundations of economics. Cambridge University Press, Cambridge

Eccles JC, Popper KR (1977) The self and its brain. An argument for interactionism. Springer International, Berlin

Earl P, Potts J (forthcoming) The market for preferences. The Cambridge Journal of Economics

Fehr E, Gächter S (2002) Altruistic punishment in humans. Nature 415: 137-140

Fehr E, Schwarz G (eds) (2002) Psychologische Grundlagen der Oekonomie. Verlag Neue Zürcher Zeitung, Zürich

Foster J (2000) Is there a role for transaction cost economics if we view firms as complex adaptive systems? Contemporary Economic Policy 18(4): 369-385

Feser E (2003) Naturalism, evolution, and Hayek's philosophy of mind. Symposium of American Institute for Economic Research on Dewey, Hayek and Embodied Cognition, Great Barrington, MA

Hayek FA (1952) The sensory order - an inquiry into the foundations of theoretical psychology. Routledge and Kegan Paul, London

Herrmann-Pillath C (1992) The brain, its sensory order and the evolutionary concept of mind: on Hayek's contribution to evolutionary epistemology. Journal of Social and Biological Structures 15(2): 147-187

Hanappi H (1994) Evolutionary economics. Avebury, Aldershot

Hodgson GM (1993) Economics and evolution: bringing life back into economics. Polity Press and Michigan University Press, Cambridge, UK Ann Arbor, MI

Kawai M (1965) Newly acquired pre-cultural behavior of the natural troop of Japanese monkeys on Koshima isles. Primates 6: 1-3

Khalil E (2003) (ed) Trust. Edward Elgar, Cheltenham

Kirchler E, Hölzl E (2003) Economic psychology. International Review of Industrial and Organizational Psychology 18: 29-80

Küppers BO (1990) Information and the origin of life. MIT Press, Cambridge MA

Lawson T (1997) Economics and reality. Routledge, London

Lim H, Park UK, Harcourt GC (eds) (2002) Editing economics. Essays in honour of Mark Perlman. Routledge, London New York

Llinas RR (2003) The neuronal basis for cognition. Symposium of American Institute for Economic Research on Dewey, Hayek and Embodied Cognition, Great Barrington, MA

Loasby BJ (1999) Knowledge, institutions and evolution in economics. Routledge, London New York

Metcalfe JS (2004) Evolutionary concepts in relation to evolutionary economics. In: Dopfer K (ed) The evolutionary foundations of economics. Cambridge University Press, Cambridge

Nelson RR, Winter SG (1982) An evolutionary theory of economic change. Harvard University Press, Cambridge, MA

Nelson RR (2002) Bringing institutions into evolutionary growth theory. Journal of Evolutionary Economics 12(1-2): 17-28

Nooteboom B (2003) Trust: forms, foundations, functions, failures and figures. Edward Elgar, Cheltenham

Perlman M (1996) The character of economic thought, economic characters, and economic institutions. The University of Michigan Press, Ann Arbor

Perlman M, McCann ChR, jr (2000) The pillars of economic understanding, 2 vols. The University of Michigan Press, Ann Arbor

Potts J (2000) The new evolutionary microeconomics: complexity, competence and adaptive behaviour. Edward Elgar, Cheltenham

Potts J (2003) Toward an evolutionary theory of Homo Oeconomicus: the concept of universal nomadism. In: Laurent J (ed) Evolutionary economics and human nature. Edward Elgar, Cheltenham

Rizzello S (2003) (ed ) Cognitive developments in economics. Routledge, London

Sanfay AG, Killing JK, Aronson JA, Hystrom LE, Cohen JD (2003) The neural basis of economic decision-making in the ultimatum game. Science 300: 1755-1758

Shackle GLS (1981) Imagination and the nature of choice. Edinburgh University Press, Edinburgh

Simon H (2004) Darwinism, altruism and economics. In: Dopfer K (ed) The evolutionary foundations of economics. Cambridge University Press, Cambridge

Sperry RW (1974) Lateral specialization in the surgically separated hemispheres. In: Schmitt FO, Worden FG (eds) The neurosciences third study program. MIT Press, Cambridge, MA 
Teuber HL (1974) Why two brains? In: Schmitt FO, Worden FG (eds) The neurosciences third study program. MIT Press, Cambridge, MA

Vromen JJ (2001) The human agent in evolutionary economics. In: Laurent J, Nightingale J (eds) Darwinism and evolutionary economics, pp 184-208. Edward Elgar, Cheltenham

deWaal FB, Johanowicz DL (1993) Modification of reconciliation behavior through social experience: an experiment with two Macaque species. Child Development 64: 897-908

Winter SG (2004) Toward an evolutionary theory of production. In: Dopfer K (ed) The evolutionary foundations of economics. Cambridge University Press, Cambridge

Witt U (1998) Imagination and leadership - the neglected dimension of an evolutionary theory of the firm. Journal of Economic Behavior and Organization 35: 161-177

Witt U (2000) Changing cognitive frames - changing organizational forms: an entrepreneurial theory of organizational development. Industrial and Corporate Change 9: 733-755

Zeier H (1980) Gehirn und Geist. Kindler, München 